\title{
Effects of Dietary Vegetable Oil on Atlantic Salmon Hepatocyte Fatty Acid Desaturation and Liver Fatty Acid Compositions
}

Running title: PUFA desaturation in salmon fed vegetable oil

Keywords: Atlantic salmon, hepatocytes, PUFA, desaturation, linseed oil, rapeseed oil, fish oil.

* To whom correspondence should be addressed at Institute of Aquaculture, University of Stirling,

\section{Douglas R. Tocher*, J.Gordon Bell, James R. Dick, and Viv O. Crampton ${ }^{1}$} Institute of Aquaculture, University of Stirling, Stirling FK9 4LA, Scotland

${ }^{1}$ Ewos Innovation, N-4335, Dirdal, Norway. Stirling FK9 4LA, Scotland. Tel.: +44-1786-467996; Fax.: +44-1786-472133.

E-mail: d.r.tocher@stir.ac.uk

3

3

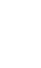

.


1 ABSTRACT: Fatty acyl desaturase activities, involved in the conversion of the $\mathrm{C}_{18}$ EFA, 18:2n-6 and

$218: 3 n-3$, to the highly unsaturated fatty acids (HUFA) 20:4n-6, 20:5n-3 and 22:6n-3, are known to be

3 under nutritional regulation. Specifically, the activity of the desaturation/elongation pathway is

4 depressed when animals, including fish, are fed fish oils rich in n-3HUFA compared to animals fed

5 vegetable oils rich in $\mathrm{C}_{18}$ EFA. The primary aims of the present study were a) to establish the relative

6 importance of product inhibition ( $n-3 H U F A)$ versus increased substrate concentration $\left(C_{18} E F A\right)$ and,

7 b) to determine whether $18: 2 n-6$ and $18: 3 n-3$ differ in their effects, on the hepatic fatty acyl

8 desaturation/elongation pathway in Atlantic salmon (Salmo salar). Smolts were fed ten experimental

9 diets containing blends of two vegetable oils, linseed (LO) and rapeseed oil (RO), and fish oil (FO) in a

10 triangular mixture design for 50 weeks. Fish were sampled after 32 and 50 weeks, lipid and fatty acid

11 composition of liver determined, fatty acyl desaturation/elongation activity estimated in hepatocytes

12 using $\left[1-{ }^{14} \mathrm{C}\right] 18: 3 \mathrm{n}-3$ as substrate, and the data subjected to regression analyses. Dietary 18:2n-6 was

13 positively correlated, and n-3HUFA negatively correlated, with lipid content of liver. Dietary 20:5n-3

14 and 22:6n-3 were positively correlated with liver fatty acids with a slope greater than unity suggesting relative retention and deposition of these HUFA. In contrast, dietary 18:2n-6 and 18:3n-3 were 16 positively correlated with liver fatty acids with a slope of less than unity suggesting metabolism via $\beta$ 17 oxidation and/or desaturation/elongation. Consistent with this, fatty acyl desaturation/elongation in 18 hepatocytes was significantly increased by feeding diets containing vegetable oils. Dietary 20:5n-3 and 19 22:6n-3 levels were negatively correlated with hepatocyte fatty acyl desaturation. At 32 weeks, 18:2n206 but not 18:3n-3, was positively correlated with hepatocyte fatty acyl desaturation activity whereas the 21 reverse was true at 50 weeks. The data indicate that both feedback inhibition through increased n22 3HUFA and decreased $\mathrm{C}_{18}$ fatty acyl substrate concentration are probably important in determining 23 hepatocyte fatty acyl desaturation activities, and that 18:2n-6 and 18:3n-3 may differ in their effects on 24 this pathway. 


\section{INTRODUCTION}

2 Virtually all animals lack $\Delta 12$ and $\Delta 15$ fatty acyl desaturases and thus are unable to biosynthesise de

3 novo the polyunsaturated fatty acids (PUFA), linoleate (18:2n-6) and linolenate (18:3n-3). Therefore,

4 these PUFA are essential fatty acids (EFA) for animals although the qualitative and quantitative

5 requirements vary among species (1). Salmonid fish such as rainbow trout (Oncorhynchus mykiss) and

6 Atlantic salmon (Salmo salar) require both 18:3n-3 and 18:2n-6 at a combined level of around 1\% of

7 the diet although the $\mathrm{C}_{18}$ EFA have no direct physiological role in fish (2). Rather their essentiality

8 derives from their conversion to the functionally active highly unsaturated fatty acids (HUFA)

9 eicosapentaenate (20:5n-3), docosahexanoate (22:6n-3) and arachidonate (20:4n-6) that are formed by

10 desaturation and elongation of the $\mathrm{C}_{18} \operatorname{EFA}(3,4)$.

11 Fatty acyl desaturase enzyme activities are known to be under nutritional regulation in mammals

12 (5) and this has also been demonstrated in fish. The activity of $\Delta 9$ desaturase (stearoyl CoA

13 dasaturase) in rainbow trout was low in starved fish and increased by feeding but was similar in fish fed

14 a diet rich in palmitic acid (16:0) compared to fish fed a standard diet containing fish oil (6). However,

15 the desaturation of 18:3n-3 and 18:2n-6 in isolated hepatocytes from Atlantic salmon was shown to be

16 greater in fish fed a diet containing a vegetable oil (a 1:1 blend of linseed and rapeseed oils) rich in

$17 \quad 18: 2 n-6$ and 18:3n-3 compared to fish fed a diet containing fish oil and thus rich in 20:5n-3 and 22:6n-3

18 (7). Several further studies have confirmed that PUFA desaturation and elongation in hepatocytes from

19 salmonid fish was increased in fish fed diets rich in $\mathrm{C}_{18}$ EFA compared to fish fed standard diets

20 containing fish oil rich in $\mathrm{C}_{20}$ and $\mathrm{C}_{22}$ HUFA (8-13). The regulation of fatty acid desaturation pathways

21 in fish is currently of great interest as there is an urgent need to replace the $\mathrm{C}_{20 / 22}$ HUFA-rich fish oils,

22 derived from potentially non-sustainable wild marine fish resources, with vegetable oils, rich in $\mathrm{C}_{18}$

23 PUFA, in the diets of aquacultured fish species (14). Demand for fish oils is rapidly outstripping

24 supply and current estimates suggest aquaculture feeds will consume more than $85 \%$ of world fish oil 
1 supplies by 2010 and so, if aquaculture is to continue to expand and supply more of the global demand

2 for fish, alternatives to fish oil must be found (15).

3 The biochemical mechanisms underpinning the nutritional regulation of the fatty acyl

4 desaturation/elongation pathway are unclear. In broad terms, feeding vegetable oils could increase the

5 activity of the PUFA desaturation/elongation pathway through two mechanisms. The pathway could be

6 simply stimulated by increased substrate $\mathrm{C}_{18}$ PUFA concentrations and/or the lack of $\mathrm{C}_{20}$ and $\mathrm{C}_{22}$ HUFA

7 could increase activity of the pathway through decreased product inhibition. Thus, the primary aims of

8 the present study are a) to establish the relative importance of decreased product inhibition and

9 increased substrate concentration and b) to determine whether 18:2n-6 and 18:3n-3 differ in their

10 effects, on the hepatic PUFA desaturation/elongation pathway in Atlantic salmon.

11 Salmon smolts were randomly stocked into ten seawater pens and, after acclimatization for two

12 weeks, were fed for 50 weeks on nine experimental diets containing various blends of two vegetable

13 oils, linseed (LO) and rapeseed oil (RO), and fish oil (FO), and a control diet containing only fish oil.

14 Fish were sampled twice, after 32 and 50 weeks of feeding the experimental diets. At each sampling

15 time, fatty acyl desaturation and elongation was estimated in isolated hepatocytes using $\left[1-{ }^{14} \mathrm{C}\right] 18: 3 \mathrm{n}-3$

16 as substrate and samples of liver were collected for analysis of lipid and fatty acid composition.

\section{MATERIALS AND METHODS}

19 Animals and diets. The experimental fish were Atlantic salmon post-smolts of initial weight $120 \pm 10$

20 g. In February 1999, hepatocyte desaturase activity was measured in a sample of fish, immediately

21 before the fish were randomly assigned to ten cages ( $5 \mathrm{~m}$ x $5 \mathrm{~m}$; 600 fish per cage) The smolts were

22 fed one of ten diets, consisting of a control diet containing FO alone and nine diets containing different

23 combinations of FO and/or vegetable oils (RO and LO) in a mixture design. Specifically, the ten diets

24 were 100\% FO, 100\% LO and 100\% RO, FO/RO (2:1 and 1:2), FO/LO (2:1 and 1:2), RO/LO (2:1 and 
$1 \quad 1: 2)$ and $\mathrm{FO} / \mathrm{RO} / \mathrm{LO}(1: 1: 1)$ forming a triangular design. The experimental diets were prepared by the

2 Ewos Technology Centre, Livingston, Scotland. Initially, the diets contained $47.0 \%$ protein, $24.1 \%$

3 lipid and $7.6 \%$ moisture ( $3 \mathrm{~mm}$ pellet) and latterly ( $6 \mathrm{~mm}$ pellet) $41.8 \%$ protein, $30.5 \%$ lipid and $6.8 \%$

4 moisture. The formulation and fatty acid compositions of the diets $(6 \mathrm{~mm}$ pellet $)$ are shown in Tables

51 and 2. All diets were formulated to satisfy the nutritional requirements of salmonid fish (16). Fish

6 were sampled twice, after feeding the experimental diets for 32 weeks (October 1999), with a final

7 sampling performed 18 weeks later in February 2000. There were no significant differences between

8 the weights of the fish on the different dietary treatments sampled after 32 weeks (ANOVA, p $>0.05$ ).

9 However, the range of weights was greater at 50 weeks $(1924 \pm 564$ to $2586 \pm 841 \mathrm{~g} ; \mathrm{n}=200)$ and

10 there were some significant differences between treatments (ANOVA, p = 0.0004). However,

11 regression analyses showed no relationship between final weight and any dietary fatty acid (including

$1216: 0,18: 1 n-9$ and total monoenes) indicating that dietary treatment was not responsible for the

13 differences. Up to 32 weeks, feed was distributed manually, but from 32 to 50 weeks the method of

14 feeding was changed from manual to automatic feeders controlled by Akvasmart pellet counters.

15 However, logistical problems at the commercial farm meant that some treatments had to be fed by hand

16 which may have affected ration and final weight to some extent. 
1 Lipid extraction and lipid class composition. Intact livers were dissected from three fish per dietary

2 treatment at each sampling point and immediately frozen in liquid nitrogen. Total lipid content of

3 livers and diet samples was determined gravimetrically after extraction by homogenization in

4 chloroform/methanol $(2: 1, \mathrm{v} / \mathrm{v})$ containing $0.01 \%$ butylated hydroxytoluene (BHT) as antioxidant,

5 basically according to Folch et al. (17). Separation of lipid classes was performed by one-dimentional,

6 double development high-performance thin-layer chromatography (HPTLC) with classes quantified by

7 charring followed by calibrated densitometry as described previously (18).

Fatty acid analysis. Fatty acid methyl esters (FAME) were prepared from total lipid by acid-

9 catalyzed transesterification using $2 \mathrm{ml}$ of $1 \% \mathrm{H}_{2} \mathrm{SO}_{4}$ in methanol plus $1 \mathrm{ml}$ toluene as described by

10 Christie (19) and FAME extracted and purified as described previously (20). FAME were separated

11 and quantified by gas-liquid chromatography (Fisons GC8600, Fisons Ltd., Crawley, U.K.) using a

$1230 \mathrm{~m}$ x $0.32 \mathrm{~mm}$ capillary column (CP wax 52CB; Chrompak Ltd., London, U.K). Hydrogen was used

13 as carrier gas and temperature programming was from $50^{\circ} \mathrm{C}$ to $180^{\circ} \mathrm{C}$ at $40^{\circ} \mathrm{C} / \mathrm{min}$ and then to $225^{\circ} \mathrm{C}$ at

$142^{\circ} \mathrm{C} / \mathrm{min}$. Individual methyl esters were identified by comparison to known standards and by reference to published data (21).

Preparation of isolated hepatocytes. Isolated hepatocytes were prepared by collagenase digestion essentially as described previously (Tocher et al., 1997) except that the sieved cells were washed twice with $20 \mathrm{ml}$ of calcium and magnesium-free Hanks balanced salt solution containing $10 \mathrm{mM}$ HEPES and $1 \mathrm{mM}$ EDTA, with the first wash also containing $1 \% \mathrm{w} / \mathrm{v}$ fatty acid-free bovine serum albumin (FAF-BSA). The hepatocytes were resuspended in $10 \mathrm{ml}$ of Medium 199 containing $10 \mathrm{mM}$ HEPES and $2 \mathrm{mM}$ glutamine. One hundred $\mu \mathrm{l}$ of cell suspension was mixed with $400 \mu \mathrm{l}$ of Trypan Blue and hepatocytes were counted and their viability assessed using a haemocytometer. One hundred $\mu l$ of the cell suspension was retained for protein determination. 
1 Assay of hepatocyte fatty acyl desaturation/elongation activities. Five $\mathrm{ml}$ of each hepatocyte

2 suspension were dispensed into a $25 \mathrm{~cm}^{2}$ tissue culture flask. Hepatocytes were incubated with 0.25

$3 \mu \mathrm{Ci}(\sim 1 \mu \mathrm{M})\left[1-{ }^{14} \mathrm{C}\right] 18: 3 \mathrm{n}-3$, added as a complex with FAF-BSA in phosphate buffered saline,

4 prepared as described previously (22). After addition of isotope the flasks were incubated at $20{ }^{\circ} \mathrm{C}$ for 2

$5 \mathrm{~h}$. The reaction was stopped, cells washed and total lipid extracted as described in detail previously

6 (23). Total lipid was transmethylated, FAME prepared as above, methyl esters separated according to

7 degree of unsaturation and chain length by argentation chromatography and autoradiography

8 perfortmed as described previously (24). Areas of silica containing individual PUFA were scraped into

9 scintillation mini-vials containing $2.5 \mathrm{ml}$ of scintillation fluid (Ecoscint A, National Diagnostics,

10 Atlanta, Georgia) and radioactivity determined in a TRI-CARB 2000CA scintillation counter (United

11 Technologies Packard, U.K.). Results were corrected for counting efficiency and quenching of ${ }^{14} \mathrm{C}$

12 under exactly these conditions.

13 Protein determination. Protein concentration in isolated hepatocyte suspensions was determined 14 according to the method of Lowry et al. (25) after incubation with $0.4 \mathrm{ml}$ of $0.25 \%$ (w/v) SDS/1M $15 \mathrm{NaOH}$ for $45 \mathrm{~min}$ at $60^{\circ} \mathrm{C}$.

16 Materials. $\left[1-{ }^{14} \mathrm{C}\right] 18: 3 \mathrm{n}-3(50-55 \mathrm{mCi} / \mathrm{mmol})$ was obtained from NEN (DuPont (U.K.) Ltd.,

17 Stevenage, U.K.). HBSS, Medium 199, HEPES buffer, glutamine, collagenase (type IV), FAF-BSA,

18 BHT and silver nitrate were obtained from Sigma Chemical Co. (Poole, U.K.). Thin-layer

19 chromatography (TLC) plates, precoated with silica gel 60 (without fluorescent indicator) were

20 obtained from Merck (Darmstadt, Germany). All solvents were HPLC grade and were obtained from

21 Fisher Scientific UK, Loughborough, England.

22 Statistical analysis. All the data are presented as means $\pm \mathrm{SD}(\mathrm{n}=3)$ unless otherwise stated. The 23 relationships between dietary fatty acid contents and growth, liver total lipid and neutral lipid contents 24 and liver fatty acid compositions, and between hepatocyte fatty acyl desaturation activity and both 
1 dietary and liver fatty acid compositions were determined by regression analyses (Prism 3, Graphpad

2 Software, Inc., San Diego, USA). In addition, the relationship between hepatocyte fatty acyl

3 desaturation activity and the source of dietary fat was examined by using stepwise multiple linear

4 regression using a mixture design (Modde 4.0, Umetri AB, Umeå, Sweden). Data from different

5 individual fish were treated as independent samples. Some data were also analyzed by one-way

6 ANOVA to determine if the overall effects of dietary treatment were significant. Percentage data and

7 data which were identified as non-homogeneous (Bartlett's test) were subjected to either arcsine or log

8 transformation before analysis. Differences were regarded as significant when $\mathrm{P}<0.05(26)$.

\section{RESULTS}

12 Dietary fatty acid compositions. The graded and systematic substitution of the three dietary oils was

13 clearly reflected in the fatty acid compositions of the ten resultant diets (Table 2). Thus, increasing

14 inclusion of RO resulted in increased proportions of 18:2n-6 and 18:1n-9 irrespective of whether it was replacing FO or LO (Table 2). Similarly, increasing inclusion of LO resulted in increased proportions of 18:3n-3 irrespective of which oil it was replacing whereas 18:2n-6 levels in diets containing LO

17 were dependent upon which oil it was replacing, increasing if FO was being replaced and decreasing if RO was being replaced (Table 2). The dietary levels of 20:5n-3 and 22:6n-3, as well as 20:4n-6, 16:0,

$1920: 1 n-9$ and 22:1n-11 were all decreased in a similarly graded manner irrespective of which vegetable

20 oil was replacing FO. In general, the levels of fatty acids were very similar in diets with the same 21 proportion of the oil that predominantly supplied that fatty acid. For example, the level of 18:3n-3 was 22 similar in diets containing the same proportion of LO. It was perhaps noteworthy that an important 23 exception to this was 18:2n-6 whose level was more dependent upon the inclusion level of all three 24 dietary oils rather than one in particular. 
1 Effects of diet on liver fatty acid compositions. The graded and systematic variation in the fatty

2 acid compositions of the ten experimental diets was reflected in the fatty acid compositions of liver

3 total lipid at both 32 and 50 weeks but was quantitatively greater at the latter time point (Table 3). In

4 particular, increasing inclusion of LO resulted in an increased proportion of $18: 3 n-3$, and inclusion of

5 RO resulted in increased proportions of $18: 2 n-6$ and $18: 1 n-9$, with the relative proportions of $20: 5 n-3$,

$622: 6 n-3$ and, to a lesser extent, 20:4n-6,16:0, 20:1n-9 and 22:1n-11 all decreased in liver lipid of fish

7 fed diets in which the FO was replaced by vegetable oils.

Effects of diet on hepatocyte fatty acid desaturation/elongation. A mixture design necessitates that

9 all three main terms (level of FO, LO and RO) are entered together or not at all. The simple linear

10 model explained a significant amount of variance (p-value of regression equation with constant plus

11 linear terms at mid and end points were $7.35 \mathrm{e}^{-11}$ and $3.196 \mathrm{e}^{-6}$ respectively, with adjusted $\mathrm{r}^{2}$ values being

120.81 and 0.58 , respectively). Adding a square term of the inclusion of RO, thus forming a binomial

13 regression equation, appreciably improved the adjusted $r^{2}$ of the regression for the end point to 0.81 and

14 had rather little effect on the value for the mid point sampling which changed to 0.82 . The use of other

15 square terms made no appreciable change to the adjusted $r^{2}$ or $p$-values. Hence the use of linear terms

16 and a square term for the inclusion of $\mathrm{RO}$ was chosen as the most appropriate regression model. Table

174 gives full details of the model, Figure 1 represents it graphically. The lack of fit term for the end

18 point data was noteworthy as the fact that the p-value was less than 0.05 suggested that, although the

19 model chosen gives a good fit explaining over $80 \%$ of the variance, there are still significant effects

20 which are not explained by the model. In contrast, since the mid point data showed a lack of fit p-value

21 of greater than 0.05 , it suggested that much of the error for these data is replicate error.

Flux through the fatty acid desaturation/elongation pathway was greater in hepatocytes from fish

23 fed diets containing vegetable oils compared to the fish fed the standard diet containing only FO and 24 the fish prior to starting the dietary treatments (Fig.1). The activities reflected the level of vegetable oil 
1 substitution, increasing linearly as the level of substitution increased. Hepatocyte fatty acid

2 desaturation/elongation was approximately 50\% higher in fish fed RO-substituted diets than in fish fed

3 LO-substituted diets. However, after 50 weeks and using the modeled levels, the lowest level of

4 hepatocyte fatty acid desaturation occurred in a wide range of RO substitution from no substitution to

5 the 50\% level (Fig.1). LO fed fish had generally higher levels than RO fed fish when the same levels

6 of substitution were compared. The changes with substitution were not linear.

7

Regression analyses of lipid and desaturation data. Regression analyses were performed to determine correlations between specific individual dietary fatty acids (18:2n-6, 18:3n-3, 20:5n-3 and 22:6n-3) or dietary fatty acid groups ( $\mathrm{C}_{18}$ PUFA and n-3HUFA) and observed liver lipid data and levels of hepatocyte fatty acid desaturation/elongation. At 32 weeks, there was no correlation between dietary fatty acids and liver total lipid contents (Table 5). However, after 50 weeks liver total lipid levels were positively correlated with dietary $18: 2 n-6$ and negatively correlated with dietary 20:5n-3, 22:6n-3 and total n-3HUFA. There were positive correlations between dietary 18:2n-6 and 18:3n-3 levels and their level in liver total lipids both at 32 and 50 weeks, with slopes all less than 1 (Table 5). The level of 20:5n-3 in liver total lipid was not correlated with dietary 20:5n-3 at 32 weeks although by 50 weeks there was a positive correlation with a slope around 1 or perhaps higher (Table 5). In contrast, the relationship between dietary and liver lipid levels of 22:6n-3 was greater than with $20: 5 n-3$ and the slope much greater than unity (Table 5 and Fig.2, panel A).

Hepatocyte fatty acid desaturation/elongation was negatively correlated with dietary 20:5n-3, 22:6n3 and total n-3HUFA both at 32 weeks and 50 weeks although the $r^{2}$ values were higher at 50 weeks (Table 5). At 32 weeks, there was a strong positive correlation between dietary 18:2n-6 and hepatocyte fatty acid desaturation whereas there was no relationship between desaturation and dietary 18:3n-3. However, at 50 weeks this situation was reversed with only $18: 3 n-3$, and not 18:2n-6, being 
1 positively correlated with hepatocyte fatty acid desaturation/elongation (Table 5). Liver fatty acid

2 compositions were less strongly related to fatty acyl desaturation/elongation. There was a strong

3 positive correlation between fatty acyl desaturation/elongation and liver 18:2n-6 levels and weak

4 negative correlations between 22:6n-3 and total n-3HUFA at 32 weeks but little of significance at 50

5 weeks (Table 5). The closer relationship between hepatocyte fatty acyl desaturation/elongation and

6 dietary fatty acid composition compared to liver fatty acid composition is evident from Fig. 2 panels B 7 and $\mathrm{C}$.

\section{DISCUSSION}

11 The present study has confirmed results from earlier studies that showed increased flux through the

12 fatty acid desaturation /elongation pathway in fish fed diets containing vegetable oils compared to fish

13 fed diets containing FO. The activities obtained with fish fed vegetable oil diets in the present study

14 were up to 4-fold higher than the activity in fish fed FO. In previous studies on salmonids in 15 freshwater, activities were up to 2.5-fold (7), 2.4-fold (12) and 2.8-fold (13) greater in fish fed VO 16 compared to fish fed FO. In salmon in seawater, 100\% replacement of FO with RO resulted in a 2.7-

17 fold increase in desaturation activity (8) whereas $100 \%$ replacement with palm oil increased the activity 18 over 10-fold (9). Similar results have been obtained in mammals. Christiansen et al. (27) fed rats diets 19 containing either sunflower oil (18:2n-6), linseed oil (18:3n-3), a combination of these vegetable oils, 20 or fish oil (n-3HUFA) and investigated the effects on liver microsomal desaturase activities. Both $\Delta 6$ 21 and $\Delta 5$ activities, as determined using 18:3n-3 and 20:3n-6 as respective substrates, were significantly 22 stimulated by feeding the diets containing vegetable oils compared to the control laboratory chow diet 23 or the fish oil diet. Microsomal $\Delta 6$ activity was significantly lower in rats fed the diet containing fish 
1 oil compared to rats fed the diets containing vegetable oils and the control chow diet when 18:2n-6 was

2 used as the substrate (27).

3 The design of the present study lends itself to statistical treatment of the resultant data by regression 4 analyses to determine potential correlations between specific dietary factors and specific outcomes. 5 This is far more illuminating than ANOVA analyses which simply indicates if there is a significant 6 difference between different diets. This was demonstrated very clearly when we sought to interpret 7 and clarify dietary effects on two potentially related parameters, growth and lipid content. Both growth and liver lipid contents showed a basically similar pattern in that there were no significant differences

9 between treatments at 32 weeks but there were significant effects after 50 weeks. There was no obvious pattern to these effects but it was possible that the two were related as lipid content is known to vary with fish weight in trout and salmon (28-30). The regression analyses showed that there was 12 absolutely no relationship between dietary fatty acids and growth. Furthermore lipid levels in the liver were not significantly related to final fish weights $\left(\mathrm{p}=0.0561, \mathrm{r}^{2}=0.3839\right)$. Thus, the primary 14 variation in final weights was almost certainly due to the change in feeding regime in the latter part of the experiment that probably affected ration (30). In contrast, the variation in liver lipid content was shown to be related to dietary fatty acids with 18:2n-6 being positively correlated indicating that it was associated with increased lipid deposition in the liver whereas n-3HUFA were negatively correlated and therefore associated with decreased liver lipid deposition. Thus regression analyses proved to be a useful tool in interpreting the effects observed in this experiment.

The strongest correlations (highly significant, and with $\mathrm{r}^{2}$ values near 1 ) were observed between 21 dietary fatty acid content and liver lipid fatty acid composition. Similar strong correlations were observed between dietary fatty acid content and muscle and liver fatty acid compositions in salmon fed 23 diets containing graded amounts of rapeseed oil (8) and palm oil (9). In the present study, the slope for 24 22:6n-3 was greater than unity which would indicate selective deposition and retention of this dietary 
1 fatty acid in liver lipid $(8,9)$. This may also be the case for $20: 5 n-3$ although the slope was actually

$21.15 \pm 0.25$. In contrast, the slopes for $18: 2 n-6$ and $18: 3 n-3$ were both less than one which suggests

3 further metabolism of these fatty acids had occurred. This could be the result of several processes

4 including catabolism for energy through $\beta$-oxidation and/or desaturation and elongation to HUFA $(8,9)$.

5 The latter is certainly a factor as evidenced by the increased desaturation and elongation observed with

6 increasing dietary content of $\mathrm{C}_{18}$ PUFA-rich vegetable oils. A similar result was observed in a previous

7 trial in salmon in which FO was replaced incrementally with RO, and where the correlations between

8 dietary fatty acid and liver fatty acid compositions gave slopes of 0.52 and 0.31 for $18: 2 n-6$ and 18:3n-

93 respectively, and 0.75 and 1.36 for $20: 5 n-3$ and 22:6n-3, respectively (8).

10 However, our primary aims in the present study were to determine the effects of specific dietary

11 fatty acids, namely 18:2n-6, 18:3n-3, 20:5n-3 and 22:6n-3 both individually and in combination $\left(\mathrm{C}_{18}\right.$

12 PUFA and n-3HUFA), on the hepatocyte fatty acyl desaturation/elongation pathway. Strong negative

13 correlations were obtained between dietary 20:5n-3, 22:6n-3 and total n-3HUFA and the total flux

14 through the fatty acid desaturation/elongation pathway at both 32 and 50 weeks. However, the most

15 significant influence on hepatocyte fatty acid desaturation was 18:2n-6 which was very strongly and

16 positively correlated at 32 weeks. In contrast, 18:3n-3 and total $C_{18}$ PUFA were not correlated with

17 fatty acid desaturation at 32 weeks but, conversely, the reverse was true at 50 weeks i.e. only 18:2n-6

18 was not correlated with desaturation. These data do not give unequivocal answers to either of our

19 hypotheses/questions. Regarding the relative importance of substrate stimulation through $\mathrm{C}_{18}$ PUFA

20 versus product inhibition through n-3HUFA, the rank order of importance changed from 18:2n-6 >

$2120: 5 n-3=22: 6 n-3>18: 3 n-3$ at 32 weeks to $22: 6 n-3=20: 5 n-3>18: 3 n-3>18: 2 n-6$ at 50 weeks using

$22 r^{2}$ values as a measure of strength of relationship. However, it could be argued that slope may be a

23 better determinant of strength of effect and, as clearly shown in Fig. 2B., a relatively small change in

24 22:6n-6 has a large effect on desaturase activity, whereas a much larger change in dietary $\mathrm{C}_{18}$ PUFA is 
1 required to produce the same magnitude of effect. This hypothesis is acknowledged as a particularly

2 challenging one to test experimentally with practical diets, as the diets with high n-3HUFA are

3 necessarily the ones with low $\mathrm{C}_{18}$ PUFA and vice versa. The mixture model (Fig.1 and Table 4) used

4 in the present study to analyze the desaturation data can be revealing, but we do not have a ready

5 explanation for the change in pattern between the mid point and end points. At the mid point, the

6 relationship was linear and, although there is a square term, it was not statistically significant and was

7 only shown to maintain consistency with terms used with the end point. In contrast, the end point data were clearly non-linear and the square term was certainly significant. This would have been very

9 difficult to predict as would the minimum level of fatty acid desaturation at the end point which was no 10 substitution to $50 \%$ RO substitution.

11 However, regarding our second hypothesis, it was evident that 18:2n-6 and 18:3n-3 indeed had 12 different effects with 18:2n-6 initially being more important, but $18: 3 n-3$ having a more significant role latterly. Therefore, the mechanism of the observed effects on fatty acyl desaturation are not clear.

14 Interestingly though, the general lack of correlation between liver fatty acid compositions and fatty acyl desaturation/elongation may be relevant. This could be due to the fact that the liver fatty acid composition was that of total lipids of which the majority was generally neutral lipid rather than membrane lipid (data not shown). However, in a study comparing the effects of dietary coconut and salmon oils in rats, the authors found no correlation between microsomal phospholipid fatty acid 19 profiles and microsomal desaturation rates (31).

In mammals, the situation regarding nutritional regulation of desaturase activities by dietary fatty acids is unclear and studies have been inconclusive. In rats, $\Delta 6$ desaturase is reported to be depressed by fasting and stimulated by EFA deficiency (5). Dietary fatty acids were also reported to affect rat $\Delta 6$ desaturase activity, with PUFA, both 20:4n-6 and 18:2n-6, being depressors, although only in 
1 increased by feeding rats a diet containing $20 \%$ safflower oil, and so rich in 18:2n-6, in comparison to

2 rats fed a diet containing $18 \%$ hydrogenated tallow and $2 \%$ safflower oil (32). Feeding the rats a diet

3 containing $10 \%$ FO with $8 \%$ hydrogenated tallow and $2 \%$ safflower oil had no significant effect on

4 intestinal $\Delta 6$ desaturase activity. Therefore, this study suggested that increased dietary substrate fatty

5 acid increased $\Delta 6$ activity whereas it's activity was not apparently depressed by increased levels of

$620: 5 n-3$ an 22:6n-3, at least with respect to desaturation of 18:2n-6. However, the activity of rat liver

$7 \Delta 6$ desaturase was higher in rats fed a diet rich in coconut oil compared to diets containing salmon oil

8 (31). As neither of these oils provided much $\mathrm{C}_{18}$ precursor fatty acids (no 18:3n-3 and only very low

9 18:2n-6), this study suggested that the presence of long-chain n-3HUFA from the salmon oil was responsible for the lower $\Delta 6$ desaturase activity rather than an effect of dietary substrate fatty acid

11 concentration. Similarly, in mice previously fed an EFA deficient diet, feeding a diet supplemented 12 with corn oil did not alter tissue desaturase activities whereas feeding a diet supplemented with fish oil 13 inhibited both $\Delta 6$ and $\Delta 5$ desaturase activities (33). Thus, studies in mammals are consistent with the 14 data obtained in the present study with salmon in suggesting that both substrate $\mathrm{C}_{18}$ PUFA content and 15 product n-3HUFA contents can be important in determining the level of fatty acid desaturation. However, it is noteworthy that the greatest stimulation of hepatocyte desaturation that we have 17 obtained in dietary trials with fish was with salmon fed a diet containing $100 \%$ palm oil, a diet that, as 18 well as being low in n-3HUFA, was also relatively low in $\mathrm{C}_{18}$ PUFA (9). Although this diet 19 formulation was not EFA-deficient, sufficient n-3HUFA to satisfy basal EFA requirements being 20 present in the fishmeal, and there were no deficiency symptoms, the fish on the $100 \%$ palm oil diet may have been bordering on EFA deficiency (9).

22 It is important to note however that, despite increased hepatic fatty acid desaturation/elongation, the 23 liver fatty acyl compositions are considerably changed by the diets in the present study. Other studies 24 have also demonstrated that replacement of large amounts of n-3HUFA-rich FO by $\mathrm{C}_{18}$ PUFA-rich 
1 vegetable oils in salmon diets significantly changes the fatty acid compositions of muscle (flesh)

2 despite increased hepatic fatty acyl desaturation (7-9,11-13). This has been apparent even in studies,

3 including this one, using diets rich in LO providing large amounts of 18:3n-3 $(7,12)$. The conclusion

4 must be that hepatic and, presumably, total body fatty acyl desaturation capacity is not sufficient to

5 convert the large amounts of dietary $18: 3 n-3$ to $20: 5 n-3$ and $22: 6 n-3$ at the rates that would be required

6 to maintain tissue n-3HUFA at levels similar to those in fish fed FO. Substrate concentration is

7 obviously not limiting as $18: 3 n-3$ is increased in both liver and muscle lipids and therefore $18: 3 n-3$ is

8 available for desaturation $(7,12)$.

9 The precise molecular mechanisms underpinning the nutritional regulation of fatty acyl desaturation

10 are unclear. In mammals, both $\Delta 6$ and $\Delta 5$ activities are depressed by fasting, and nutrients such as

11 glucose, fructose and glycerol are reportedly depressors whereas protein is an activator (5). Regulation

12 by nutritional state and specific nutrients is likely mediated by hormones. Certainly, insulin is known

13 to activate both $\Delta 6$ and $\Delta 5$ desaturases (34). Cyclic AMP and glucagon (probably via cAMP) block

14 the increase in $\Delta 6$ activity at refeeding, and epinephrine suppresses both $\Delta 6$ and $\Delta 5$ via activation of $\beta$ -

15 receptors (34). However, the involvement of protein kinases and phosphorylation in the regulation of

16 fatty acyl desaturases has not been elucidated. Glucocorticoids and other steroids are also depressors of

$17 \Delta 6$ and $\Delta 5$ desaturases suggesting another mechanism, transcriptional control, in the regulation of

18 desaturase activity. Recently, the cloning of PUFA desaturase enzymes from both mammals (35-37)

19 and fish $(38,39)$ has enabled gene expression to be studied. In mice fed $10 \%$ corn oil rich in 18:2n-6,

20 the mRNA abundance and hepatic $\Delta 6$ activity were respectively $50 \%$ and $70 \%$ lower than those in

21 mice fed $10 \%$ triolein, an EFA-deficient diet (35). The levels of hepatic mRNA for both $\Delta 6$ and $\Delta 5$

22 desaturases in rats fed either $10 \%$ safflower oil (18:2n-6) or menhaden oil (n-3HUFA) were only $25 \%$

23 of those in rats fed a fat-free diet or a diet containing triolein (36). The liver mRNA level of a putative 
$1 \Delta 6$ desaturase cloned from rainbow trout was significantly higher in trout fed LO compared to trout fed

2 FO (39). These results imply transcriptional regulation of desaturases in response to dietary fatty acids.

3 The regulation of hepatic gene transcription by fatty acids, particularly PUFA, is being increasingly

4 studied (40). PUFA can potentially affect gene transcription by a number of direct and indirect

5 mechanisms including changes in membrane composition, eicosanoid production, oxidant stress,

6 nuclear receptor activation or covalent modification of specific transcription factors (41). PUFA are

7 now known to bind and directly influence the activities of a variety of transcription factors such as

8 peroxisome proliferator-activated receptors (PPARs), which have in turn been shown to be critical

9 regulators of a growing list of genes involved in lipid homeostatic processes (42). In rodents,

10 peroxisomal proliferators, which also activate PPARs, are known to up-regulate fatty acyl $\Delta 6, \Delta 5$ and

$11 \Delta 9$ desaturases (43-45). PPAR genes have been identified in Atlantic salmon and plaice $(46,47)$ and the

12 peroxisomal proliferator, clofibrate, increased the desaturation of $20: 5 n-3$ in rainbow trout (48).

13 However, these data do not exclude the possibility that fatty acids may also influence desaturase more

14 directly at a membrane level through alterations in fluidity or membrane microenvironments.

\section{REFERENCES}

1. Holman, R.T. (1986) Control of Polyunsaturated Fatty Acids in Tissue Lipids, J. Am. Coll. Nutr. 5 , 19 183-211.

2. Sargent, J. R., Tocher, D. R., and Bell, J. G. (2002) The Lipids, in Fish Nutrition (Halver, J. E. and Hardy, R.W, eds.), 3rd Edition, Ch.4, pp. 181-257, Academic Press, San Diego. Criteria for Essential Fatty Acids, J. Appl. Ichthyol. 11, 183-198. 
4. Sargent, J.R., Bell, J.G., McEvoy, L., Tocher, D.R., and Estevez, A. (1999) Recent Developments in the Essential Fatty Acid Nutrition of Fish, Aquaculture 177, 191-199.

5. Brenner, R.R. (1981) Nutritional and Hormonal Factors Influencing Desaturation of Essential Fatty Acids, Prog. Lipid Res. 20, 41-47.

6. Tocher, D.R., Bell, J.G., and Sargent, J.R. (1996) Induction of $\Delta$ 9-Fatty Acyl Desaturation in Rainbow Trout (Oncorhynchus mykiss) Liver by Dietary Manipulation, Comp. Biochem. Physiol. $113 B, 205-212$

7. Bell, J.G., Tocher, D.R., Farndale, B.M., Cox, D.I., McKinney, R.W., and Sargent, J.R. (1997) The Effect of Dietary Lipid on Polyunsaturated Fatty Acid Metabolism in Atlantic Salmon (Salmo salar) Undergoing Parr-Smolt Transformation, Lipids 32, 515-525.

8. Bell, J.G., McEvoy, J., Tocher, D.R., McGhee, F., Campbell, P.J., and Sargent, J.R. (2001) Replacement Fish Oil with Rape Seed Oil in Diets of Atlantic Salmon (Salmo salar) Affects Tissue Lipid Compositions and Fatty Acid Metabolism, J. Nutr. 131, 1535-1543.

9. Bell, J.G., Henderson, R.J., Tocher, D.R., McGhee, F., Dick, J.R., Porter, A., Smullen, R., and Sargent, J.R. (2002) Substituting Fish Oil with Crude Palm Oil in the Diet of Atlantic Salmon (Salmo salar) Affects Tissue Fatty Acid Compositions and Hepatic Fatty Acid Metabolism, $J$. Nutr.132, 222-230.

10. Buzzi, M., Henderson, R.J., and Sargent, J.R. (1996) The Desaturation and Elongation of Linolenic Acid and Eicosapentaenoic Acid by Hepatocytes and Liver Microsomes from Rainbow Trout (Oncorhyncus mykiss) Fed Diets Containing Fish Oil or Olive Oil, Biochim. Biophys. Acta 1299, 235-244.

11. Tocher, D.R., Bell, J.G., Dick, J.R., and Sargent, J.R. (1997) Fatty Acyl Desaturation in Isolated Hepatocytes from Atlantic Salmon (Salmo salar): Stimulation by Dietary Borage Oil Containing $\gamma$ Linolenic Acid, Lipids 32, 1237-1247. 
1 12. Tocher, D.R., Bell, J.G., Henderson, R.J., McGhee, F., Mitchell, D., and Morris, P.C. (2000) The Effect of Dietary Linseed and Rapeseed Oils on Polyunsaturated Fatty Acid Metabolism in Atlantic Salmon (Salmo salar) Undergoing Parr-Smolt Transformation, Fish. Physiol. Biochem. 23, 59-73.

13. Tocher, D.R., Bell, J.G., MacGlaughlin, P., McGhee, F., and Dick, J.R. (2001) Hepatocyte Fatty

14. Sargent J.R., and Tacon, A. (1999) Development of Farmed Fish: A Nutritionally Necessary Dietary Vegetable Oil, Comp. Biochem Physiol. 130, 257-270.

15. Barlow, S. (2000) Fishmeal and Oil: Sustainable Feed Ingredients for Aquafeeds, Global Aquacult. Alternative to Meat, Proc. Nutr. Soc. 58, 377-383. Advocate 4, 85-88.

16. U.S. National Research Council. (1993) Nutrient Requirements of Fish, National Academy Press, Washington D.C.

17. Folch, J., Lees, M., and Sloane-Stanley, G.H. (1957) A Simple Method for the Isolation and Purification of Total Lipids from Animal Tissues, J. Biol. Chem. 226, 497-509.

18. Henderson, R.J., and Tocher, D.R. (1992) Thin-Layer Chromatography, in Lipid Analysis : A Practical Approach (Hamilton, R.J., and Hamilton, S., eds.), pp. 65-111, Oxford University Press, Oxford.

19. Christie, W.W. (1982) Lipid Analysis, 2nd Edition. P.207, Pergamon Press, Oxford.

20. Tocher, D.R., and Harvie, D.G. (1988) Fatty Acid Compositions of the Major Phosphoglycerides from fish Neural Tissues : (n-3) and (n-6) Polyunsaturated Fatty Acids in Rainbow Trout (Salmo gairdneri, L.) and Cod (Gadus morhua) Brains and Retinas, Fish Physiol. Biochem. 5, 229-239.

21. Ackman, R.G. 1980. Fish Lipids, part 1, in Advances in Fish Science and Technology (Connell, J.J., ed.), pp. 87-103, Fishing News Books, Farnham, U.K. 
1 22. Ghioni, C., Tocher, D.R., and Sargent, J.R. (1997) The Effect of Culture on Morphology, Lipid and Fatty Acid composition, and Polyunsaturated Fatty Acid Metabolism of Rainbow Trout (Oncorhynchus mykiss) Skin Cells, Fish Physiol. Biochem. 16, 499-513.

23. Tocher, D.R., Sargent, J.R., and Frerichs, G.N. (1988) The Fatty Acid Compositions of Established Fish Cell Lines After Long-Term Culture in Mammalian Sera, Fish Physiol. Biochem. 5, 219-227.

24. Wilson, R., and Sargent, J.R. (1992) High Resolution Separation of Polyunsaturated Fatty Acids by Argentation Thin-Layer Chromatography, J. Chromatogr. 623, 403-407.

25. Lowry, O.H., Rosebrough, N.J., Farr, A.L., and Randall, R.J. (1951) Protein Measurement With the Folin Phenol Reagent, J. Biol. Chem. 193, 265-275.

26. Zar, J.H. (1984) Biostatistical Analysis, 2nd Edition. Prentice-Hall, Englewood Cliffs.

27. Christiansen, E.N., Lund, J.S., Rortveit, T., and Rustan, A.C. (1991) Effect of Dietary n-3 and n-6 Fatty Acids on Fatty Acid Desaturation in Rat Liver, Biochim. Biophys. Acta 1082, 57-62.

28. Storebakken, T., Hung, S.S.O., Calvert, C.C., and Plisetskaya, E.M. (1991) Nutrient Partitioning in Rainbow Trout at Different Feeding Rates, Aquaculture 96, 191-203.

29. Sheehan, E.M., O’Connor, T.P., Sheehy, P.J.A., Buckley, D.J., and FitzGerald, R. (1996) Effect of Dietary Fat Intake on the Quality of Raw and Smoked Salmon, Irish J. Agric. Fd. Res. 35, $37-42$.

30. Johansen, S.J.S., and Jobling, M. (1998) The Influence of Feeding Regime on Growth and Slaughter Ttraits of Cage-Reared Atlantic Salmon, Aquaculture Internat. 6, 1-17.

31. Ulmann, L., Bouziane, M., Mimouni, V., Belleville, J., and Poisson, J.-P. (1992) Relationship Between Rat Liver Microsomal $\Delta 6$ and $\Delta 5$ Desaturase Activities and Fatty Acid Composition: Comparative Effects of Coconut and Salmon Oils During Protein Restriction, J. Nutr. Biochem. $3,188-193$.

32. Garg, M.L., Keelan, M., Thomson, A.B.R., and Clandinin, M.T. (1992) Desaturation Linoleic 
1 acid in the Small Bowel is Increased by Short-Term Fasting and by Dietary Content of Linoleic Acid, Biochim. Biophys. Acta 1126, 17-25.

3 33. Raz, A., Kamin-Belsky, N., Przedecki, F., and Obukowicz, M.G. (1997) Fish Oil Inhibits $\Delta 6$

4 Desaturase Activity In Vivo: Utility in a Dietary Paradigm to Obtain Mice Depleted of

5 Arachidonic Acid, J. Nutr. Biochem. 8, 558-565.

6 34. Cook, H.W. (1996) Fatty Acid Desaturation and Chain Elongation in Eukaryotes, in

7 Biochemistry of Lipids, Lipoproteins and Membranes (Vance, D.E., and Vance, J.E., eds.), pp. 129-152, Elsevier, Amsterdam.

35. Cho, H. P., Nakamura, M.T., and Clarke, S.D. (1999a) Cloning, Expression, and Nutritional Regulation of the Mammalian s-6 Desaturase, J. Biol. Chem. 274, 471-477.

36. Cho, H. P., Nakamura, M.T., and Clarke, S.D. (1999b) Cloning, Expression, and Fatty Acid

37. Aki, T., Shimada, Y., Inagaki, K., Higashimoto, H., Kawamoto, S., Shigeta, S., Ono, K., and

39. Seiliez, I., Panseat, S., Kaushik, S., and Bergot, P. (2001) Cloning, Tissue Distribution and Nutritional Regulation of a $\Delta 6$-Desaturase-Like Enzyme in Rainbow Trout, Comp. Biochem. Physiol. 130B, 83-93.

40. Jump, D. B., and Clarke, S.D. (1999) Regulation of Gene Expression by Dietary Fat, Annu. Rev. Nutr. 19, 63-90. 
1 41. Jump, D. B., Thelen, A., Ren, B., and Mater, M. (1999) Multiple Mechanisms for Polyunsaturated Fatty Acid Regulation of Hepatic Gene Transcription, Prostaglandins Leukotrienes Essent. Fatty Acids 60, 345-349.

42. Jump, D. B. (2002) The Biochemistry of n-3 Polyunsaturated Fatty Acids, J. Biol. Chem. 277,

43. Kawashima, Y., Musoh , K., and Kozuka, H. (1990) Peroxisome Proliferators Enhance Linoleic Acid Metabolism in Rat Liver. Increased Biosynthesis of $\omega 6$ Ppolyunsaturated Fatty Acids, J. Biol. Chem. 265, 9170-9175.

44. Gronn, M., Christensen, E., Hagve, T. -A., and Christophersen, B.O. (1992) Effects of Clofibrate Feeding on Essential Fatty Acid Desaturation and Oxidation in Isolated Rat Liver Cells, Biochim. Biophys. Acta 1123, 170-176.

45. Alegret, M., Cerqueda, E., Ferramdo, R., Vazquez, M., Sanchez, R.M., Adzet, T., Merlos, M., and Laguna, J.C. (1995) Selective Modification Rat Hepatic Microsomal Fatty Acid chain Elongation and Desaturation by Fibrates: Relationship With Peroxisomal Proliferation, Br. J. Pharmacol. 114, $1351-1358$.

46. Ruyter, B. Andersen, O., Dehli, A., Ostlund Farrants, A.-K., Gjoen, T., and Thomassen, M.S. (1997) Peroxisome Proliferator Activated Receptors in Atlantic Salmon (Salmo salar): Effects on PPAR Transcription and acyl-CoA Oxidase Activity in Hepatocytes by Peroxisome Proliferators and Fatty Acids, Biochim. Biophys. Acta 1348, 331-338.

47. Leaver, M. J., Wright, J., and George, S.G. (1998) A Peroxisome Proliferator Activated Receptor Gene from the Marine Flatfish, the Plaice (Pleuronectes platessa), Mar. Env. Res. 46, 75-79.

48. Tocher, D.R., and Sargent, J.R. (1993) No Relationship Between Morphology Changes and Metabolism of $\alpha$-Linolenate and Eicosapentaenoate in Rainbow Trout (Oncorhynchus mykiss) Astroglial Cells in Primary Culture, Comp. Biochem. Physiol. 106C, 211-219. 
Legends to Figures:

FIG. 1. Mixture contour plot of levels of hepatocyte fatty acid desaturation at mid and end point sampling. Vertices of the triangles represent $100 \%$ of the added oil from fish oil (upper vertex), linseed oil (lower left vertex), or rapeseed oil (lower right vertex). Contour lines represent the modeled levels of fatty acid desaturation using the model shown in Table 4.

FIG. 2. Regression analyses showing relationships between fatty acid compositions and hepatocyte fatty acyl desaturation/elongation after 50 weeks. A, relationships between dietary and liver total lipid levels of 18:3n-3 and 22:6n-3; B, relationships between dietary $\mathrm{C}_{18}$ PUFA and 22:6n-3 levels and hepatocyte fatty acid desaturation/elongation; $C$, relationships between levels of $\mathrm{C}_{18}$ PUFA and 22:6n-3 in liver total lipid and hepatocyte fatty acid desaturation. 


\section{Mid Point}

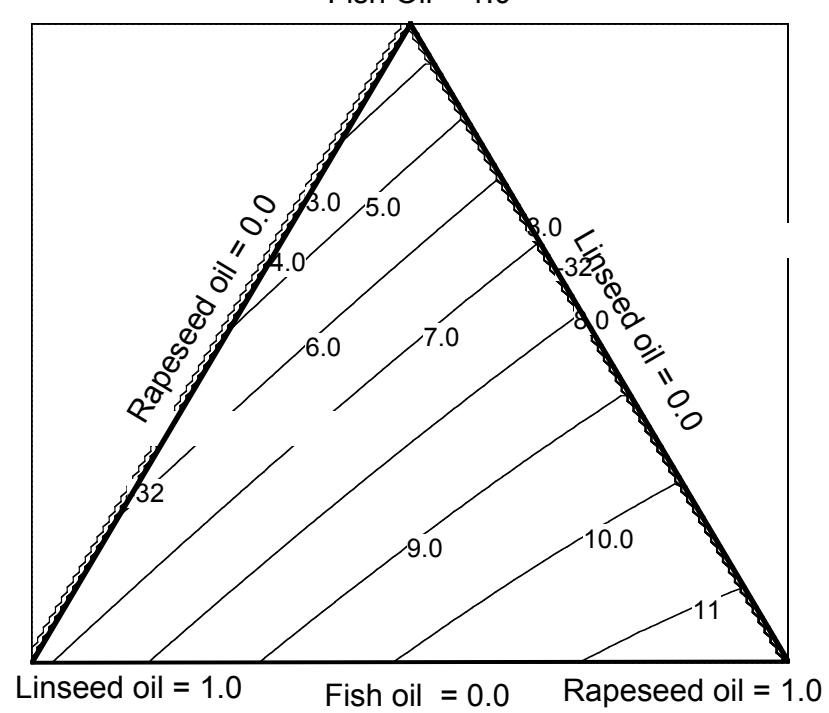

2. End Point

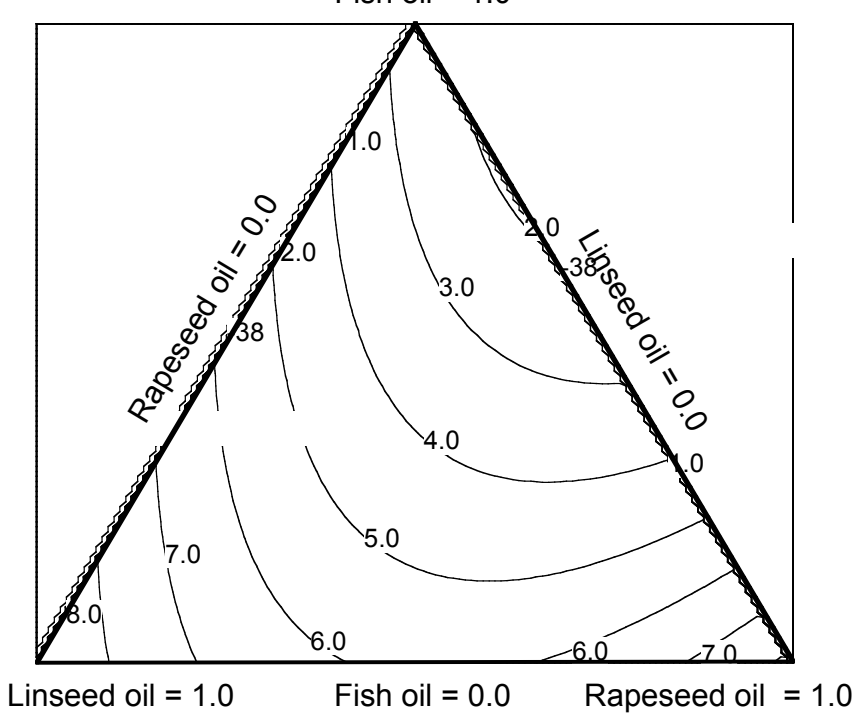



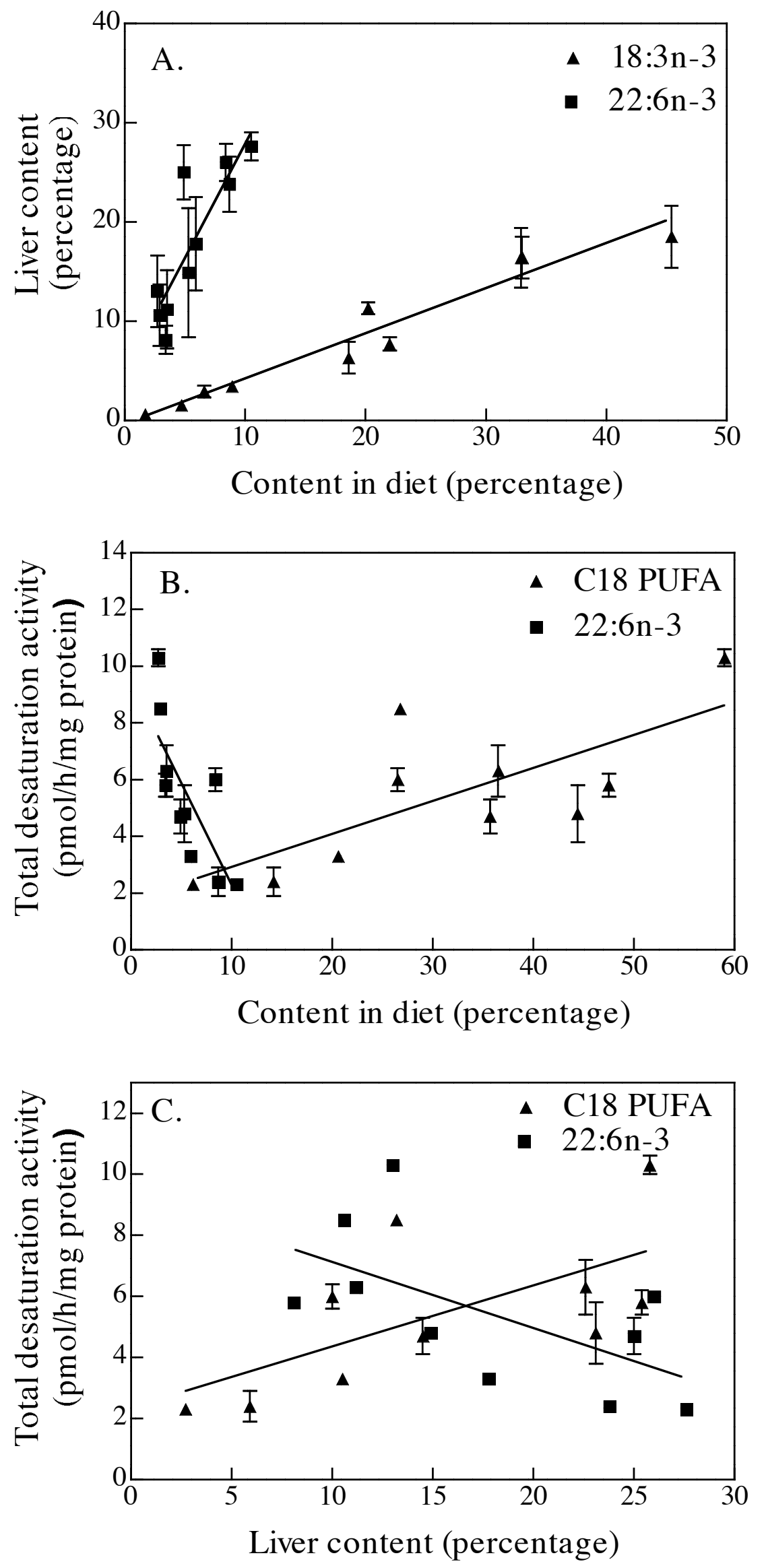
Table 1. Feed composition (g/100g).

\begin{tabular}{|c|c|c|c|c|c|c|c|c|c|c|}
\hline Component & $\mathrm{FO}$ & $\begin{array}{c}\mathrm{FO} / \mathrm{LO} \\
(2: 1) \\
\end{array}$ & $\begin{array}{c}\mathrm{FO} / \mathrm{LO} \\
(1: 2) \\
\end{array}$ & LO & $\begin{array}{c}\mathrm{FO} / \mathrm{RO} \\
(2: 1) \\
\end{array}$ & $\begin{array}{c}\text { FO:RO } \\
(1: 2)\end{array}$ & RO & $\begin{array}{c}\mathrm{LO} / \mathrm{RO} \\
(2: 1) \\
\end{array}$ & $\begin{array}{c}\mathrm{LO} / \mathrm{RO} \\
(1: 2) \\
\end{array}$ & $\begin{array}{c}\mathrm{FO} / \mathrm{LO} / \mathrm{RO} \\
(1: 1: 1) \\
\end{array}$ \\
\hline Fishmeal $^{1}$ & 53.8 & 53.8 & 53.8 & 53.8 & 53.8 & 53.8 & 53.8 & 53.8 & 53.8 & 53.8 \\
\hline Soya (Hi Pro) ${ }^{2}$ & 7.6 & 7.6 & 7.6 & 7.6 & 7.6 & 7.6 & 7.6 & 7.6 & 7.6 & 7.6 \\
\hline Wheat $^{3}$ & 14 & 14 & 14 & 14 & 14 & 14 & 14 & 14 & 14 & 14 \\
\hline Fish oil $^{4}$ & 23.6 & 15.7 & 7.9 & 0 & 15.7 & 7.9 & 0 & 0 & 0 & 7.9 \\
\hline Rapeseed oil $^{5}$ & 0 & 0 & 0 & 0 & 7.9 & 15.7 & 23.6 & 7.9 & 15.7 & 7.9 \\
\hline Linseed oil ${ }^{5}$ & 0 & 7.9 & 15.7 & 23.6 & 0 & 0 & 0 & 15.7 & 7.9 & 7.9 \\
\hline Micronutrients $^{6}$ & 1 & 1 & 1 & 1 & 1 & 1 & 1 & 1 & 1 & 1 \\
\hline
\end{tabular}

${ }^{1}$ Norseameal, London, England.

${ }^{2}$ Grosvenor Grain, Perth, Scotland.

${ }^{3}$ Stewarts of Larbert, Larbert, Scotland.

${ }^{4}$ United Fish Products, Aberdeen, Scotland.

${ }^{5}$ Meade-King Robinson \& Co., Liverpool, England.

${ }^{6}$ Vitamins, minerals and carotenoid pigment (Roche Products, Heanor, England to specification by Ewos Ltd., Bathgate, Scotland) 
Table 2. Fatty acid compositions of the ten experimental diets. (Values are weight $\%$ of total fatty acids)

\begin{tabular}{|c|c|c|c|c|c|c|c|c|c|c|}
\hline Fatty acid/diet & $\mathrm{FO}$ & $\begin{array}{c}\text { FO/LO } \\
(2: 1) \\
\end{array}$ & $\begin{array}{c}\mathrm{FO} / \mathrm{LO} \\
(1: 2) \\
\end{array}$ & $\mathrm{LO}$ & $\begin{array}{c}\mathrm{FO} / \mathrm{RO} \\
(2: 1) \\
\end{array}$ & $\begin{array}{c}\mathrm{FO} / \mathrm{RO} \\
(1: 2) \\
\end{array}$ & $\mathrm{RO}$ & $\begin{array}{c}\mathrm{LO} / \mathrm{RO} \\
(2: 1) \\
\end{array}$ & $\begin{array}{c}\mathrm{LO} / \mathrm{RO} \\
(1: 2) \\
\end{array}$ & $\begin{array}{c}\text { FO/LO/RO } \\
(1: 1: 1) \\
\end{array}$ \\
\hline $14: 0$ & 5.6 & 3.6 & 2.2 & 1.1 & 3.7 & 2.3 & 1.2 & 1.2 & 1.1 & 1.9 \\
\hline $16: 0$ & 13.6 & 11.6 & 9.6 & 7.6 & 11.7 & 9.4 & 7.2 & 7.7 & 7.6 & 8.8 \\
\hline 18:0 & 2.5 & 2.9 & 3.4 & 3.6 & 2.3 & 2.0 & 1.9 & 3.0 & 2.4 & 2.7 \\
\hline Total saturates ${ }^{1}$ & 24 & 20.2 & 16.7 & 13.2 & 19.9 & 15.5 & 11.4 & 13.0 & 10.9 & 14.9 \\
\hline $16: 1 n-7$ & 5.5 & 3.5 & 2.2 & 1.0 & 3.8 & 2.5 & 1.3 & 1.2 & 1.3 & 1.9 \\
\hline $18: 1 n-9$ & 15.1 & 16.1 & 16.4 & 16.6 & 26.7 & 37.5 & 48.3 & 25.9 & 36.9 & 28.8 \\
\hline $20: 1 n-9$ & 9.6 & 6.0 & 3.7 & 1.9 & 6.5 & 4.6 & 3.0 & 2.2 & 2.6 & 3.5 \\
\hline $22: 1 n-11$ & 13.2 & 8.2 & 4.8 & 2.3 & 8.3 & 5.4 & 2.7 & 2.6 & 2.7 & 4.3 \\
\hline Total monounsaturates ${ }^{2}$ & 44.3 & 34.6 & 27.6 & 22.1 & 46.2 & 50.6 & 55.6 & 32.3 & 45.5 & 39.0 \\
\hline $18: 2 n-6$ & 4.5 & 7.9 & 11.4 & 13.6 & 9.5 & 14.0 & 17.9 & 14.6 & 16.3 & 13.7 \\
\hline $20: 4 n-6$ & 0.6 & 0.4 & 0.3 & 0.2 & 0.5 & 0.3 & 0.2 & 0.2 & 0.2 & 0.2 \\
\hline Total n-6 PUFA ${ }^{3}$ & 5.5 & 8.7 & 11.8 & 13.9 & 10.4 & 14.6 & 18.1 & 15.0 & 16.8 & 14.1 \\
\hline $18: 3 n-3$ & 1.7 & 18.6 & 33.0 & 45.4 & 4.7 & 6.6 & 8.9 & 32.9 & 20.2 & 22.0 \\
\hline $18: 4 n-3$ & 2.8 & 1.8 & 1.0 & 0.5 & 1.9 & 1.1 & 0.4 & 0.4 & 0.4 & 0.9 \\
\hline $20: 4 n-3$ & 0.8 & 0.6 & 0.3 & 0.1 & 0.6 & 0.4 & 0.1 & 0.2 & 0.1 & 0.2 \\
\hline $20: 5 n-3$ & 7.3 & 5.3 & 3.3 & 1.8 & 5.7 & 3.9 & 1.9 & 2.2 & 2.1 & 3.1 \\
\hline $22: 5 n-3$ & 1.2 & 1.0 & 0.6 & 0.3 & 1.1 & 0.6 & 0.3 & 0.4 & 0.4 & 0.5 \\
\hline $22: 6 n-3$ & 10.5 & 8.4 & 5.3 & 2.7 & 8.7 & 5.9 & 2.9 & 3.4 & 3.5 & 4.9 \\
\hline Total n-3 PUFA & 24.5 & 35.8 & 43.6 & 50.8 & 22.8 & 18.6 & 14.5 & 39.6 & 26.7 & 31.5 \\
\hline$n-3 / n-6$ & 4.4 & 4.1 & 3.7 & 3.7 & 2.2 & 1.3 & 0.8 & 2.6 & 1.6 & 2.2 \\
\hline
\end{tabular}

${ }^{1}$ Includes 10:0, 12:0, 17:0, 20:0 and 22:0. Includes 14:1, 17:1, 20:1n-7, 22:1n-9 and 24:1. ${ }^{3}$ Includes 18:3n-6, 20:2n-6, 20:3n-6 and 22:5n-6. 
Table 3. Fatty acid compositions (percentage of total fatty acids by weight) of total lipid of liver from Atlantic salmon (Salmo salar L.) fed the experimental diets for 50 weeks.

\begin{tabular}{|c|c|c|c|c|c|c|c|c|c|c|}
\hline & FO & $\mathrm{FO} / \mathrm{LO}(2: 1)$ & $\mathrm{FO} / \mathrm{LO}(1: 2)$ & LO & $\mathrm{FO} / \mathrm{RO}(2: 1)$ & $\mathrm{FO} / \mathrm{RO}(1: 2)$ & RO & $\mathrm{LO} / \mathrm{RO}(2: 1)$ & $\mathrm{LO} / \mathrm{RO}(1: 2)$ & $\mathrm{FO} / \mathrm{LO} / \mathrm{RO}(1: 1: 1)$ \\
\hline $14: 0$ & $1.9 \pm 0.2$ & $1.3 \pm 0.1$ & $1.2 \pm 0.2$ & $0.7 \pm 0.1$ & $1.4 \pm 0.2$ & $1.0 \pm 0.1$ & $0.8 \pm 0.2$ & $0.7 \pm 0.1$ & $0.6 \pm 0.0$ & $1.0 \pm 0.0$ \\
\hline $16: 0$ & $14.1 \pm 2.0$ & $12.9 \pm 0.8$ & $8.2 \pm 0.8$ & $9.8 \pm 1.2$ & $11.1 \pm 1.2$ & $8.8 \pm 1.4$ & $9.2 \pm 2.0$ & $6.6 \pm 1.3$ & $6.4 \pm 1.5$ & $11.7 \pm 1.2$ \\
\hline 18:0 & $4.3 \pm 0.3$ & $4.6 \pm 0.3$ & $3.9 \pm 1.1$ & $6.3 \pm 0.6$ & $4.5 \pm 0.3$ & $3.7 \pm 0.2$ & $4.5 \pm 0.4$ & $4.3 \pm 0.8$ & $3.8 \pm 0.3$ & $5.0 \pm 0.6$ \\
\hline Total saturated $^{1}$ & $20.5 \pm 2.1$ & $19.0 \pm 0.7$ & $13.4 \pm 1.7$ & $16.7 \pm 1.8$ & $17.1 \pm 1.4$ & $13.7 \pm 1.5$ & $14.5 \pm 2.4$ & $11.6 \pm 2.1$ & $10.8 \pm 1.8$ & $17.8 \pm 1.7$ \\
\hline $16: 1 n-7$ & $2.4 \pm 0.2$ & $2.0 \pm 0.3$ & $2.6 \pm 1.6$ & $1.6 \pm 1.0$ & $2.1 \pm 0.1$ & $1.7 \pm 0.3$ & $1.8 \pm 0.7$ & $1.9 \pm 0.6$ & $0.9 \pm 0.1$ & $1.1 \pm 0.1$ \\
\hline $18: 1 n-9$ & $14.9 \pm 2.3$ & $15.1 \pm 1.3$ & $21.8 \pm 6.3$ & $22.2 \pm 5.3$ & $22.7 \pm 2.5$ & $32.2 \pm 6.4$ & $40.1 \pm 3.8$ & $33.0 \pm 2.6$ & $32.8 \pm 5.0$ & $17.4 \pm 3.0$ \\
\hline $18: 1 \mathrm{n}-7$ & $2.8 \pm 0.1$ & $2.0 \pm 0.1$ & $2.1 \pm 0.4$ & $1.3 \pm 0.2$ & $2.9 \pm 0.1$ & $3.1 \pm 0.3$ & $3.0 \pm 0.3$ & $1.5 \pm 1.3$ & $2.7 \pm 0.2$ & $1.9 \pm 0.2$ \\
\hline $20: 1 \mathrm{n}-9$ & $6.2 \pm 0.8$ & $4.3 \pm 1.0$ & $4.2 \pm 0.7$ & $2.5 \pm 0.2$ & $6.2 \pm 0.6$ & $6.0 \pm 0.5$ & $5.4 \pm 1.0$ & $3.9 \pm 0.2$ & $4.1 \pm 0.5$ & $3.1 \pm 0.5$ \\
\hline $22: 1$ & $2.2 \pm 0.4$ & $1.5 \pm 0.4$ & $1.4 \pm 0.3$ & $0.7 \pm 0.2$ & $1.6 \pm 0.6$ & $1.3 \pm 0.2$ & $0.9 \pm 0.1$ & $0.8 \pm 0.1$ & $0.9 \pm 0.2$ & $0.9 \pm 0.2$ \\
\hline $24: 1 n-9$ & $1.2 \pm 0.2$ & $0.9 \pm 0.1$ & $0.5 \pm 0.3$ & $0.5 \pm 0.1$ & $0.8 \pm 0.0$ & $0.6 \pm 0.3$ & $0.6 \pm 0.1$ & $0.4 \pm 0.1$ & $0.6 \pm 0.1$ & $1.2 \pm 0.2$ \\
\hline Total monoenes ${ }^{2}$ & $30.1 \pm 3.3$ & $26.2 \pm 2.4$ & $33.1 \pm 8.3$ & $29.0 \pm 6.8$ & $36.7 \pm 3.4$ & $45.4 \pm 7.5$ & $52.3 \pm 5.0$ & $41.8 \pm 2.1$ & $42.3 \pm 5.5$ & $25.8 \pm 3.9$ \\
\hline $18: 2 n-6$ & $2.1 \pm 0.1$ & $3.7 \pm 0.6$ & $6.7 \pm 0.8$ & $7.3 \pm 1.0$ & $4.4 \pm 0.6$ & $7.6 \pm 1.0$ & $9.8 \pm 0.8$ & $9.0 \pm 1.2$ & $11.3 \pm 1.0$ & $6.8 \pm 1.0$ \\
\hline $20: 2 n-6$ & $0.7 \pm 0.1$ & $1.0 \pm 0.1$ & $1.4 \pm 0.3$ & $1.4 \pm 0.3$ & $1.4 \pm 0.1$ & $2.1 \pm 0.1$ & $2.2 \pm 0.3$ & $1.7 \pm 0.1$ & $1.9 \pm 0.1$ & $1.2 \pm 0.1$ \\
\hline $20: 3 n-6$ & $0.2 \pm 0.0$ & $0.2 \pm 0.0$ & $0.2 \pm 0.0$ & $0.3 \pm 0.1$ & $0.2 \pm 0.0$ & $0.4 \pm 0.1$ & $0.8 \pm 0.2$ & $0.4 \pm 0.1$ & $0.6 \pm 0.0$ & $0.4 \pm 0.1$ \\
\hline $20: 4 n-6$ & $2.2 \pm 0.2$ & $1.9 \pm 0.1$ & $0.9 \pm 0.7$ & $0.7 \pm 0.2$ & $1.7 \pm 0.2$ & $1.1 \pm 0.4$ & $0.7 \pm 0.2$ & $0.4 \pm 0.0$ & $0.8 \pm 0.2$ & $2.1 \pm 0.5$ \\
\hline $22: 5 n-6$ & $0.4 \pm 0.0$ & $0.3 \pm 0.0$ & $0.2 \pm 0.1$ & $0.2 \pm 0.0$ & $0.3 \pm 0.0$ & $0.2 \pm 0.1$ & $0.1 \pm 0.0$ & $0.1 \pm 0.0$ & $0.1 \pm 0.0$ & $0.3 \pm 0.0$ \\
\hline Total n-6 PUFA ${ }^{3}$ & $5.7 \pm 0.1$ & $7.1 \pm 0.5$ & $9.4 \pm 0.6$ & $10.0 \pm 1.4$ & $8.2 \pm 0.5$ & $11.5 \pm 0.4$ & $13.7 \pm 1.2$ & $11.7 \pm 1.2$ & $14.8 \pm 0.8$ & $10.7 \pm 0.6$ \\
\hline $18: 3 n-3$ & $0.6 \pm 0.0$ & $6.3 \pm 1.6$ & $16.4 \pm 2.1$ & $18.5 \pm 3.1$ & $1.5 \pm 0.3$ & $2.9 \pm 0.6$ & $3.4 \pm 0.2$ & $16.4 \pm 3.0$ & $11.3 \pm 0.6$ & $7.7 \pm 0.7$ \\
\hline $20: 3 n-3$ & $0.2 \pm 0.0$ & $1.5 \pm 0.3$ & $3.9 \pm 0.8$ & $3.7 \pm 0.8$ & $0.5 \pm 0.0$ & $0.8 \pm 0.1$ & $0.7 \pm 0.1$ & $3.7 \pm 0.8$ & $2.1 \pm 0.2$ & $1.3 \pm 0.1$ \\
\hline $20: 4 n-3$ & $1.4 \pm 0.2$ & $1.5 \pm 0.1$ & $1.9 \pm 0.3$ & $2.2 \pm 0.4$ & $1.3 \pm 0.2$ & $0.9 \pm 0.1$ & $0.7 \pm 0.2$ & $2.1 \pm 0.3$ & $2.0 \pm 0.3$ & $1.5 \pm 0.4$ \\
\hline $20: 5 n-3$ & $10.3 \pm 0.3$ & $9.0 \pm 0.7$ & $4.6 \pm 1.8$ & $5.2 \pm 0.7$ & $7.6 \pm 0.4$ & $4.8 \pm 2.0$ & $3.2 \pm 0.8$ & $3.1 \pm 0.5$ & $4.1 \pm 1.2$ & $8.0 \pm 1.2$ \\
\hline $22: 5 n-3$ & $3.3 \pm 0.5$ & $2.9 \pm 0.4$ & $1.9 \pm 0.2$ & $1.2 \pm 0.1$ & $2.9 \pm 0.1$ & $2.0 \pm 0.5$ & $0.8 \pm 0.2$ & $1.0 \pm 0.2$ & $1.0 \pm 0.3$ & $1.8 \pm 0.2$ \\
\hline $22: 6 n-3$ & $27.6 \pm 1.4$ & $26.0 \pm 1.9$ & $14.9 \pm 6.5$ & $13.0 \pm 3.6$ & $23.8 \pm 2.8$ & $17.8 \pm 4.7$ & $10.6 \pm 3.1$ & $8.1 \pm 1.4$ & $11.2 \pm 3.9$ & $25.0 \pm 2.7$ \\
\hline Total n-3 PUFA & $43.7 \pm 1.2$ & $47.6 \pm 1.7$ & $44.1 \pm 6.3$ & $44.3 \pm 6.7$ & $37.9 \pm 2.4$ & $29.4 \pm 6.5$ & $19.5 \pm 4.2$ & $35.0 \pm 3.1$ & $32.1 \pm 4.5$ & $45.6 \pm 2.7$ \\
\hline$n-3 / n-6$ & $7.7 \pm 0.3$ & $6.7 \pm 0.4$ & $4.7 \pm 0.6$ & $4.4 \pm 0.1$ & $4.6 \pm 0.6$ & $2.6 \pm 0.7$ & $1.4 \pm 0.3$ & $3.0 \pm 0.1$ & $2.2 \pm 0.4$ & $4.3 \pm 0.5$ \\
\hline
\end{tabular}

${ }^{1}$ Includes $15: 0$ present at up to $0.5 \% ;{ }^{2}$ Includes $16: 1 \mathrm{n}-9$ and $20: 1 \mathrm{n}-7$ each present at up to $0.5 \% ;{ }^{3}$ Includes $18: 3 \mathrm{n}-6$ and $22: 4 \mathrm{n}-6$ each present at up to $0.5 \%$;

${ }^{4}$ Includes 18:4n-3 and 22:4n-3 each present at up to $0.5 \%$; FO, fish oil; LO, linseed oil; PUFA, polyunsaturated fatty acids; RO, rapeseed oil. 
Table 4. Coefficients of the model shown in Figure 1 and related statistical evaluation.

\begin{tabular}{|c|c|c|c|c|c|c|}
\hline \multirow[b]{2}{*}{ Term } & \multicolumn{3}{|c|}{ Mid point } & \multicolumn{3}{|c|}{ End point } \\
\hline & Value & Standard error & p-value & Value & Standard error & p-value \\
\hline \multicolumn{7}{|c|}{ Coefficients of the model } \\
\hline Constant & 8.49 & 1.321 & $8.28 \mathrm{e}^{-7}$ & 2.894 & 1.17 & 0.02 \\
\hline Proportion of FO & -5.178 & 1.433 & $1.27 \mathrm{e}^{-3}$ & -0.278 & 1.269 & 0.828 \\
\hline Proportion of LO & -1.725 & 1.433 & 0.239 & 6.248 & 1.269 & $4.11 \mathrm{e}^{-5}$ \\
\hline Proportion of RO & 6.903 & 1.797 & $7.08 \mathrm{e}^{-4}$ & -5.97 & 1.592 & $8.95 \mathrm{e}^{-4}$ \\
\hline$(\text { Proportion of RO) })^{2}$ & -3.779 & 2.169 & 0.093 & 11.429 & 1.922 & $2.82 \mathrm{e}^{-6}$ \\
\hline Statistical evaluation & \multicolumn{3}{|c|}{ Mid point } & \multicolumn{3}{|c|}{ End point } \\
\hline Number of cases & \multicolumn{3}{|c|}{30} & \multicolumn{3}{|c|}{30} \\
\hline $\begin{array}{l}\text { Degrees of freedom, } \\
\text { regression }\end{array}$ & \multicolumn{3}{|c|}{3} & \multicolumn{3}{|c|}{3} \\
\hline $\begin{array}{l}\text { Degrees of freedom, } \\
\text { residual }\end{array}$ & \multicolumn{3}{|c|}{26} & \multicolumn{3}{|c|}{26} \\
\hline $\mathrm{F}$ value & \multicolumn{3}{|c|}{45.8} & \multicolumn{3}{|c|}{43.5} \\
\hline Adjusted $r^{2}$ & \multicolumn{3}{|c|}{0.823} & \multicolumn{3}{|c|}{0.815} \\
\hline p-value regression & \multicolumn{3}{|c|}{$1.61 \mathrm{e}^{-10}$} & \multicolumn{3}{|c|}{$2.78 \mathrm{e}^{-10}$} \\
\hline Lack of Fit $p$ value & \multicolumn{3}{|c|}{0.075} & \multicolumn{3}{|c|}{$6.03 \mathrm{e}^{-8}$} \\
\hline $\begin{array}{l}\text { Residual standard } \\
\text { deviation }\end{array}$ & \multicolumn{3}{|c|}{1.21} & \multicolumn{3}{|c|}{1.07} \\
\hline
\end{tabular}

FO, fish oil, LO, linseed oil, RO, rapeseed oil. 
Table 5. Correlation (regression) analyses ( $\mathrm{r}^{2}$ slope values and significance) for dietary fatty acids and liver total lipid content, dietary and liver fatty acid compositions, and hepatocyte fatty acyl desaturation activity and dietary and liver fatty acid compositions

\begin{tabular}{|c|c|c|c|c|c|c|}
\hline \multirow[b]{2}{*}{ Fatty acid } & \multicolumn{3}{|c|}{32 weeks } & \multicolumn{3}{|c|}{50 weeks } \\
\hline & $r^{2}$ & slope & $\overline{\text { significance }}$ & $\mathrm{r}^{2}$ & slope & significance \\
\hline \multicolumn{7}{|c|}{ A. Diet v. Liver total lipid } \\
\hline $18: 2 n-6$ & 0.04 & $0.02 \pm 0.04$ & 0.5714 & 0.45 & $0.41 \pm 0.16$ & 0.0326 \\
\hline $18: 3 n-3$ & 0.05 & $0.01 \pm 0.01$ & 0.5203 & 0.09 & $0.05 \pm 0.06$ & 0.3984 \\
\hline Total C18 PUFA & 0.02 & $0.00 \pm 0.01$ & 0.6690 & 0.19 & $0.07 \pm 0.05$ & 0.2048 \\
\hline $20: 5 n-3$ & 0.01 & $-0.02 \pm 0.08$ & 0.8435 & 0.44 & $-0.87 \pm 0.35$ & 0.0371 \\
\hline $22: 6 n-3$ & 0.01 & $-0.01 \pm 0.06$ & 0.7575 & 0.46 & $-0.61 \pm 0.23$ & 0.0300 \\
\hline Total n-3HUFA & 0.01 & $-0.01 \pm 0.03$ & 0.8033 & 0.44 & $-0.31 \pm 0.12$ & 0.0359 \\
\hline \multicolumn{7}{|l|}{ B. Diet v.Liver } \\
\hline $18: 2 n-6$ & 0.89 & $0.49 \pm 0.06$ & $<0.0001$ & 0.91 & $0.67 \pm 0.07$ & $<0.0001$ \\
\hline $18: 3 n-3$ & 0.93 & $0.28 \pm 0.03$ & $<0.0001$ & 0.94 & $0.46 \pm 0.04$ & $<0.0001$ \\
\hline $20: 5 n-3$ & 0.19 & $0.35 \pm 0.26$ & 0.2052 & 0.73 & $1.15 \pm 0.25$ & 0.0017 \\
\hline $22: 6 n-3$ & 0.51 & $1.11 \pm 0.38$ & 0.0204 & 0.75 & $2.30 \pm 0.47$ & 0.0012 \\
\hline \multicolumn{7}{|c|}{ C. Diet v. Desaturation } \\
\hline $18: 2 n-6$ & 0.80 & $0.63 \pm 0.11$ & 0.0005 & 0.34 & $0.37 \pm 0.18$ & 0.0785 \\
\hline $18: 3 n-3$ & 0.00 & $-0.003 \pm 0.07$ & 0.9645 & 0.43 & $0.12 \pm 0.05$ & 0.0398 \\
\hline Total C18 PUFA & 0.05 & $0.04 \pm 0.06$ & 0.5588 & 0.54 & $0.12 \pm 0.04$ & 0.0162 \\
\hline $20: 5 n-3$ & 0.50 & $-1.07 \pm 0.38$ & 0.0223 & 0.58 & $-1.04 \pm 0.31$ & 0.0106 \\
\hline $22: 6 n-3$ & 0.50 & $-0.73 \pm 0.26$ & 0.0230 & 0.59 & $-0.72 \pm 0.21$ & 0.0097 \\
\hline Total n-3HUFA & 0.50 & $-0.39 \pm 0.14$ & 0.0219 & 0.58 & $-0.38 \pm 0.11$ & 0.0102 \\
\hline \multicolumn{7}{|c|}{ D. Liver v. Desaturation } \\
\hline $18: 2 n-6$ & 0.83 & $1.23 \pm 0.2$ & 0.0002 & 0.29 & $0.48 \pm 0.27$ & 0.1106 \\
\hline $18: 3 n-3$ & 0.002 & $-0.03 \pm 0.24$ & 0.8935 & 0.34 & $0.22 \pm 0.11$ & 0.0751 \\
\hline Total C18 PUFA & 0.13 & $0.21 \pm 0.19$ & 0.3025 & 0.43 & $0.20 \pm 0.08$ & 0.0408 \\
\hline $20: 5 n-3$ & 0.26 & $-0.96 \pm 0.57$ & 0.1328 & 0.26 & $-0.52 \pm 0.31$ & 0.1311 \\
\hline $22: 6 n-3$ & 0.42 & $-0.43 \pm 0.18$ & 0.0436 & 0.38 & $-0.22 \pm 0.10$ & 0.0586 \\
\hline Total n-3HUFA & 0.47 & $-0.32 \pm 0.12$ & 0.0285 & 0.28 & $-0.16 \pm 0.09$ & 0.1136 \\
\hline
\end{tabular}

Correlation (regression) analyses of A. Dietary fatty acid contents and fish final weights (growth), B. Dietary and liver fatty acid composions, C. Dietary fatty acid composition and hepatocyte fatty acyl desaturation and, D. Liver fatty acid composition and hepatocyte fatty acyl desaturation. 